\title{
Similarity After Goodman
}

\author{
Lieven Decock • Igor Douven
}

Published online: 26 June 2010

(C) The Author(s) 2010. This article is published with open access at Springerlink.com

\begin{abstract}
In a famous critique, Goodman dismissed similarity as a slippery and both philosophically and scientifically useless notion. We revisit his critique in the light of important recent work on similarity in psychology and cognitive science. Specifically, we use Tversky's influential set-theoretic account of similarity as well as Gärdenfors's more recent resuscitation of the geometrical account to show that, while Goodman's critique contained valuable insights, it does not warrant a dismissal of similarity.
\end{abstract}

\section{Formal Modes of Similarity}

For much of the twentieth century, both philosophical and psychological theorizing about similarity has been dominated by the geometrical model of similarity (see, e.g., Carnap 1928/1967; Coombs 1954; Shepard 1958; Torgerson 1958, 1965). This model is clearly implicit in Goodman's critique of similarity. We start by giving a summary statement of the model and by briefly discussing its shortcomings, as pointed at in Goodman's critique and, a bit later, in Tversky's (1977). We then discuss Tversky's and Gärdenfors's accounts of

\footnotetext{
L. Decock

Faculty of Philosophy, VU University Amsterdam, De Boelelaan 1105, 1081 HV Amsterdam, The Netherlands e-mail: lb.decock@ph.vu.nl

I. Douven $(\varangle)$

Faculty of Philosophy, University of Groningen,

Oude Boteringerstraat 52, 9712 GL Groningen, The Netherlands

e-mail: igor.douven@rug.nl
} 
similarity, showing that these do not share the shortcomings of the geometrical account.

\subsection{The Geometrical Model of Similarity}

According to the geometrical model, similarity relations can be represented by means of a so-called metric similarity space. A metric space is a pair $\langle X, \delta\rangle$ with $X$ a set of points and $\delta$ a metric (or distance function) on $X$, that is, $\delta: X \times X \rightarrow \mathbb{R}_{0}^{+}$with, for all $a, b, c \in X$ :

Minimality:

Symmetry:

$$
\delta(a, b) \geqslant 0, \text { and } \delta(a, b)=0 \text { iff } a=b ;^{1}
$$

Triangle Inequality:

$\delta(a, b)=\delta(b, a)$

When interpreted in the context of similarity, $\delta(a, b)$ can be thought of as measuring the degree of dissimilarity of the objects represented by $a$ and $b$ in the space. ${ }^{2}$

Patently, this approach to similarity yields a formally exact explication of similarity. As a further advantage we note that it enables one to model comparative similarity judgments in an entirely straightforward manner: objects $a$ and $b$ are at least as similar (more similar) to each other as (than) objects $c$ and $d$ iff $\delta(a, b) \leqslant(<) \delta(c, d)$. And, by defining a fixed threshold value for distances in the metric similarity space, we can also model absolute similarity judgments: objects $a$ and $b$ are similar to each other iff their distance does not exceed a certain threshold value $\mathbf{t} \in \mathbb{R}^{+}$, that is, iff $\delta(a, b) \leqslant \mathbf{t}$. Finally, while the model is clearly very unspecific, leaving entirely open the question of what determines the mapping of objects onto points in the similarity space, this at the same time makes it very general. For instance, it is compatible with different views about which faculties or processes underlie people's similarity judgments.

Unfortunately, the geometrical model is seriously flawed. As Goodman was the first to note, ${ }^{3}$ similarity judgments are highly dependent on context:

[C]omparative judgments of similarity often require not merely selection of relevant properties but a weighting of their relative importance, and variation in both relevance and importance can be rapid and enormous. Consider baggage at an airport checking station. The spectator may notice shape, size, color, material, and even make of luggage; the pilot

\footnotetext{
${ }^{1}$ This condition is slightly stronger than the condition that goes by the same name in Tversky (1977: 328): $\delta(a, b) \geqslant \delta(a, a)=0$. A function satisfying the latter condition together with Symmetry and Triangle Inequality is, contrary to what Tversky suggests, not guaranteed to be a metric, but only a pseudo-metric.

${ }^{2}$ In the following we will conflate the names of objects with the names of the points representing the objects in the space.

${ }^{3}$ At least in the literature; we suppose that the point is too obvious not to have been noted independently by others.
} 
is more concerned with weight, and the passenger with destination and ownership. Which pieces are more alike than others depends not only upon what properties they share, but upon who makes the comparison, and when.... Circumstances alter similarities. (Goodman 1972: 445)

This context-sensitivity is clearly a problem for the geometrical model, inasmuch as this model contains no parameter that could be made to vary with context.

In addition to this, Tversky (1977) presents empirical evidence casting doubt on the tenability of the basic axioms of metric spaces when interpreted in terms of similarity. Already Minimality poses a problem, given that it entails reflexivity of similarity, meaning that the degree of similarity of $a$ to itself is equal to the degree of similarity of $b$ to itself, for all objects $a$ and $b$. This is a problem because, in psychological experiments, the probability of judging two stimuli identical turned out not to be the same for all kinds of identical stimuli. Triangle Inequality is not corroborated by the data either; in some experiments, in which objects were similar in entirely different respects, violation of this condition occurred (cf. Tversky and Gati 1982). Nevertheless, Tversky's critique concerns in the first place Symmetry. He demonstrated that people's similarity judgments are in many cases not symmetrical. For example, experiments reveal that typically North Korea is judged to be more similar to China than China is to North Korea. This provides a direct refutation of the geometrical model.

\subsection{Tversky's Set-theoretic Approach}

The deficiencies of the geometrical model led Tversky to develop a settheoretical approach to similarity based on feature matching. The approach presupposes a domain $D=\{a, b, c, \ldots\}$ of objects that are not characterized by points in a geometrical space, but by a set of features; for example, an orange may be represented by a set of features $A=\{$ round, orange, medium-sized, juicy, ripe, ... $\}$. Similarity is then defined in terms of certain set-theoretical relations obtaining (or failing to obtain) between sets of features representing different objects.

To be more exact, on Tversky's proposal the similarity relation must fulfil several conditions. The most important of these are the matching condition and the monotonicity condition. The former says that how similar two objects are is a function strictly of the set of their shared features and the two respective sets of their distinctive features (the set of features that belong to the first but not to the second, and the set of features that belong to the second but not to the first). Formally, for all $a, b \in D$,

$$
S(a, b)=F(A \cap B, A \backslash B, B \backslash A),
$$


with $S$ being the similarity relation, $F$ some ternary real-valued function, $A$ the features of $a$, and $B$ the features of $b$. According to the monotonicity condition,

$$
\begin{array}{ll}
S(a, b) \geqslant S(a, c) \quad \text { if } & \text { (i) } A \cap C \subseteq A \cap B ; \\
& \text { (ii) } A \backslash B \subseteq A \backslash C ; \\
& \text { (iii) } B \backslash A \subseteq C \backslash A .
\end{array}
$$

In the above, " $\geqslant$ " can be replaced by ">" just in case at least one of the three inclusion relations is proper; that is to say, an object $b$ is more similar to an object $a$ than an object $c$ is if either the features $a$ and $c$ share are among the ones shared by $a$ and $b$, or the features not shared by $a$ and $b$ are among the ones not shared by $a$ and $c$, or both. An important consequence of this is that similarity increases with the addition of common features and/or the deletion of distinctive features. In addition to the matching and monotonicity conditions, there are also three more technical conditions, which Tversky terms independence, solvability, and invariance; we refer the reader to Tversky (1977: 351) for the definitions.

Tversky then proves a representation theorem to the effect that if $S$ fulfils the foregoing conditions, then there is a similarity scale $s$ and a nonnegative scale $f$ such that, for all $a, b, c, d \in D$,

(i) $s(a, b) \geqslant s(c, d)$ iff $S(a, b) \geqslant S(c, d)$;

(ii) $s(a, b)=\theta f(A \cap B)-\alpha f(A \backslash B)-\beta f(B \backslash A)$, for some $\theta, \alpha, \beta \geqslant 0$;

(iii) $f$ and $s$ are interval scales.

This representation of the similarity relation has been named "the contrast model": similarity is expressed as a "contrast" between the measures of the common and the distinctive features. ${ }^{4}$

It is worth noticing that the representation theorem does not characterize a unique similarity scale $s$ but rather a family of such scales. For instance, if $\alpha=\beta=0$ but $\theta>0$, we have a similarity relation that only depends on the shared features of two objects. If, on the other hand, $\theta=0$ but $\alpha, \beta>0$, we have a similarity relation that only depends on the features not shared by the two objects. In addition to this, a function $f$ must be fixed. This function is most naturally construed as a salience function. Certain sets of common or distinctive features contribute more to the similarity scale than others and are thus more salient in comparisons of objects.

Already from the above summary statement of Tversky's model it should be clear that the model explains swiftly the experimentally established asymmetry in people's similarity judgments. For nothing in the model requires that $\alpha=\beta$ : the task presented to the participants in an experiment may be what

\footnotetext{
${ }^{4}$ Besides the contrast model, there are other models based on feature matching. An interesting alternative is the ratio model, according to which $S(a, b)=f(A \cap B) /[f(A \cap B)+\alpha f(A \backslash B)+$ $\beta f(B \backslash A)$ ]; see Tversky (1977: 333).
} 
Tversky calls "directional." Therefore, if the complement sets of features do not have the same measure (i.e., if $f(A \backslash B) \neq f(B \backslash A)$ ), we may have the asymmetrical $S(a, b) \neq S(b, a)$.

Tversky's approach can also easily deal with the context-dependence of similarity judgments that Goodman brought to bear against the geometrical model. For, first, the set of features that represents an object will in general be only a subset of the set of all the properties the object has. For the representation of the objects in the domain, only a limited set of features is considered relevant, and the selection of these features is relative to certain interests and purposes, which may vary with context. Second, even given a fixed set of salient features, the salience of these features relative to one another may change from one context to the next. Finally, the selection of the domain may be context-sensitive. That this may have implications for the similarity judgments is nicely illustrated by Tversky's extension effect (Tversky 1977: 343); see also Medin et al. (1993: 361). The effect is that if all the objects under consideration share a feature, it tends to become neutral in the comparison, but if an object not sharing the feature is added to the comparison task, the feature at once becomes salient. The activation of this feature may shift the similarity judgments between the former objects that do share the feature. For instance, adding a non-European country to a comparison task that so far involved only European countries may lead people to revise their previous similarity judgments regarding European countries.

Still, if Medin et al. (1993) are right, similarity judgments may be even more sensitive to context than was anticipated by Tversky and than his contrast model can handle, at least as it stands. In Tversky's view, it is possible at least in experimental set-ups to prevent context shifts from occurring, and therefore also to select a priori a similarity scale appropriate for the context of the experiment relative to which the subjects' similarity judgments can be assessed. But, backed by experimental findings, Medin et al. doubt that this is correct. As they say:

It is natural to assume that, to constrain similarity comparison appropriately, the representation of each of the constituent terms must be rigid (i.e., context-insensitive). In contrast, our observations suggest that the effective representations of the constituents are determined in the context of the comparison, not prior to it. It is as if the two terms were dancers: Each dancer may have a repertoire of stylistic preferences, but the actual performance depends on an interaction between the two. For asymmetrical comparisons, the "base dancer" takes the lead and the "target dancer" follows. The result is appropriately constrained even though the constituents are quite flexible. (Medin et al. 1993: 275)

In other words, the "respect" in which two objects (constituent terms) are similar is, or at least may be, selected in the process of comparing the objects, and is not necessarily fixed in advance. Accordingly, Medin et al. put forward a double explanation of the asymmetry of certain similarity judgments. As on 
Tversky's account, the similarity scale $s_{C}$ can be asymmetrical in context $C$. In addition, however, the order of presentation of the objects may determine which object "takes the lead." Reversing this order may create a different context. Hence, in a particular situation, the similarity judgment $S(a, b)$ may be based on $s_{C}(a, b)$, while the similarity judgment $S(b, a)$ is based on $s_{C^{\prime}}(b, a)$, with $C^{\prime} \neq C$. This difference in context can either be a difference in the domain, a difference in selected features, a difference in salience of the selected features, a difference in the contrast parameters $\theta, \alpha, \beta$, or a combination of the foregoing. However, putting Medin et al.'s point of critique this way, it is already obvious that the critique does not so much undermine Tversky's model, but rather suggests a slight modification of it, to wit, that the contextinsensitive similarity scale $s$ be replaced by a set of scales $\left\{s_{C} \mid C\right.$ a context $\}$, containing a scale $s_{C}$ for each context $C$.

Thus, like the geometrical model, Tversky's account defines similarity in a formally precise way. Unlike the geometrical model, however, Tversky's account (at least if slightly modified in the just-designated way) also enjoys considerable empirical support. In particular, it is able to explain the registered asymmetries in, as well as the context-sensitivity of, people's similarity judgments. So, supposing Tversky's account to be correct, there is no reason for worries that similarity is a slippery and scientifically suspect notion.

\subsection{Gärdenfors's Refined Geometrical Approach}

The main stumbling blocks for the old geometrical model, we saw, were the fact that it is unable to account for asymmetries in people's similarity judgments as well as for the context-sensitivity of such judgments. Recently, a number of authors have tried to overcome these problems by modifying the geometrical model. Specifically, these authors argue that, while similarity can be thought of as having a geometrical structure indeed, the proponents of the geometrical model have been mistaken in presuming that a single general metric similarity space underlies all similarity judgements. To rectify this mistake, a contextualized geometrical notion of similarity has been developed in recent psychological and cognitive science literature, most notably in Gärdenfors's Conceptual Spaces (2000). ${ }^{5}$

The point of departure of these endeavors has been the plausible claim that general similarity judgments are not meaningful, and that, first, objects

\footnotetext{
${ }^{5}$ Clark (1993) is an interesting earlier attempt to resuscitate the geometrical approach. We have based our account on Gärdenfors because it is more explicitly contextual, and also simpler in that it takes similarity spaces as basic, whereas Clark takes a similarity relation as basic, and then construes similarity spaces out of that by means of a technique known as "multidimensional scaling." Other modifications of the geometrical model are to be found in Krumhansl (1978), Holman (1979), Nosofsky (1991), and Johannesson (2000). These models handle the asymmetry of proximity judgments by explaining such judgments in terms of both the distance function in similarity space and another factor, such as density (Krumhansl), bias (Holman, Nosofsky), or prominence (Johannesson).
} 
can only be similar in certain respects, and second, different respects can be salient in different contexts. They then argue that the relevant notion of respect can be cashed out by reference to conceptual spaces. For example, if one compares objects with respect to color, one invokes a color space. Another, perhaps even simpler, example of a conceptual space is a three-dimensional Euclidean space with a Euclidean metric serving to represent proximity in visual space. ${ }^{6}$ Still further examples are the one-dimensional temporal space, the two-dimensional auditory space, olfactory and taste spaces (see Clark 1993: 140-146), and even shape spaces (see Gärdenfors 2000: 94-98). In short, one crucial idea is that, instead of a general similarity space, we have at our avail a multitude of conceptual spaces (or similarity spaces). The second crucial idea is that in each context typically only a subset of all these spaces is activated, to wit, those corresponding to the respects that are salient in the given context.

Together, these ideas provide a precise definition of similarity that meets the objections regarding context-sensitivity that Goodman and others have levelled against the original geometrical model. In addition to this, it furnishes a plausible explanation of the asymmetry effects noted by Tversky and Medin et al., for it is reasonable to hold that if, for instance, subjects are asked how similar $a$ is to $b$, this may activate a different set of conceptual spaces than when they are asked how similar $b$ is to $a$. Normally, this new set of conceptual spaces will consist of the initial conceptual spaces with rescaled axes (see Gärdenfors 2000: 132ff).

We thus appear to have at least two formally precise definitions of similarity, both of which can accommodate not only the context-sensitivity that Goodman rightly mounted against the geometrical model in its original form, but also all further known experimental results concerning similarity judgments. It is important to note here that we intend our discussion to be confined to Tversky's and Gärdenfors's accounts insofar as they aim to model similarity relations between single objects. As accounts of similarity relations between what in the literature are sometimes called "multipart scenes" (which typically involve multiple objects or figures), both Tversky's and Gärdenfors's account may fare less well; see Goldstone and Son (2005: 22ff) and references given there. A second point to note is that, for present purposes, there is no need to determine which of the said accounts (if either) is the correct account of similarity. ${ }^{7}$ The important observation here is that either account helps to give the lie to Goodman's contention that similarity cannot be adequately defined and therefore is a slippery notion.

\footnotetext{
${ }^{6}$ One often finds the term "phenomenal visual space" for this space (e.g., in Shepard 2001). For a discussion of the metaphysical status of this space, see Decock (2006).

${ }^{7}$ In fact, we believe that one should be open to the possibility that both accounts capture different senses of "similarity," much in the way in which, for instance, the different recent probabilistic explications of the notion of coherence may capture slightly different senses of "coherence" (see Meijs 2005).
} 


\section{Similarity and the Property Structure}

A second important element in Goodman's critique of the notion of similarity concerns the relation between similarity and the property structure. Goodman's sixth stricture (p. 441) states that similarity between objects does not suffice to define properties, and the seventh stricture (p. 443) states that similarity cannot be equated with, or measured in terms of, possession of common properties. As we hope to make clear, a closer look at the relation between similarity and the property structure, informed by recent work on the notion of quasi-analysis alongside Tversky's and Gärdenfors's accounts of similarity, seriously undermines Goodman's main arguments.

Goodman has a straightforward argument against Leibniz's proposal that similarity can be equated with the sharing of properties. Famously, Leibniz construed identity as the sharing of all properties and, analogously, similarity as the sharing of at least one property. According to Leibniz, two objects are similar just in case they share at least one property (Leibniz 1923: A64 107/P 13). Goodman argues that this proposal is useless, because any two objects share at least one property, so that similarity is a universal relationeverything is similar to everything else-and similarity claims are thus utterly uninformative. Indeed, if we go along with Goodman (1972) in conceiving of properties as classes of objects, then, in view of the set-theoretic pairing axiom, every two objects always share the membership of at least one class. The problem equally arises for a triadic similarity relation " $x$ is more similar to $y$ than to $z$," or a tetradic similarity relation " $x$ and $y$ are more similar than $z$ and $t$ are," if these are explicated in terms of the sharing of properties (e.g., if it is said that $a$ is more similar to $b$ than to $c$ in case $a$ and $b$ share more properties than $a$ and $c$ do). For it is easily shown that in a universe with $n$ objects, every two objects share $2^{n-2}$ properties; given an infinite universe, every pair of objects even has an infinite number of common properties.

Straightforward though the argument may be, it hinges on two implicit assumptions that deserve a closer look. The first assumption is extensionalism, that is, the thesis that every property is fully characterized by a set of objects. The second is that every set of objects determines a property. As to extensionalism, it is somewhat surprising, in view of the remarkable comeback of intensional logic in the 1960s, that Goodman rejects out of hand intensional definitions of properties, saying only that "the idea of measuring similarity or anything else in terms of number of intensional entities need hardly to be taken seriously" (p. 444). As to the second assumption, extensionalists could give it up by restricting the class of properties to "important" (Goodman 1972: 444) or "natural" (Quine 1969: 114), or (with a slightly different motivation) "projectible" (Goodman 1965: 357) properties. According to Goodman (1972), this is an unattractive option, since "importance is a highly volatile matter, varying with every shift of context or interest" (p. 444). However, if we accept that context-sensitivity need not be a problem, the characterization of important or natural properties reduces to clarifying how a particular context determines a structure of natural properties. This contextual property structure can thus 
determine a contextual similarity relation. There are no a priori reasons why this could not be done. In short, both of Goodman's implicit assumptions are debatable, and hence so is his critique of Leibniz's characterization of similarity that rests on them.

In a sense, however, these are just preliminary skirmishes, for Goodman's critique is not in the first place directed against defining similarity on the basis of properties, but rather the other way around, against the construction of a property structure on the basis of the similarity relation. Goodman's doctoral thesis (1941/1990) as well as his The Structure of Appearance (1977) are based on Carnap's project of quasi-analysis as formulated in Der logische Aufbau der Welt (1967). In the Aufbau, a complete structure of properties in the world is reconstructed on the basis of one single (phenomenal) relation Rs, which stands for "recollection of similarity" (1967: 127). A reflexive and symmetric similarity relation ${ }^{8}$ between two events (elementary experiences) is defined as " $x$ Rs $y$ or $y$ Rs $x$." Quasi-analysis is a mathematical procedure to define properties (or quasi-properties) on the basis of such a similarity relation. For a given "similarity structure," that is, a set $S$ with a reflexive and symmetric relation $\sim$ defined on it, we can look for a set of extensional properties, that is, classes of elements of $S$, such that every two similar objects in $S$ have at least one property in common, and that dissimilar objects do not share any property. Carnap's proposal in the Aufbau ( $\S 70-73, \S \S 80-81, \S 97, \S 104)$ was to take similarity circles ${ }^{10}$ as properties, that is, maximal sets of objects that are all pairwise similar.

Goodman's (1977) main reasons for thinking that properties cannot be defined in terms of similarity are twofold, to wit, the companionship difficulty-which, he believes, "obviously constitutes a serious defect in the proposed method" (p. 117)—and the difficulty of imperfect community, which is even said to be "virtually disastrous to the proposed construction" (ibid.). The former difficulty stems from the fact that properties obtained by quasianalysis are maximal sets of similar objects. This poses a problem because if, as may occur, some property applies only to a subset of a maximal set of similar objects, this property cannot be recovered by quasi-analysis. To explain the second and allegedly more serious difficulty, consider Goodman's (1972: 442) case of the two-colored discs (see also his 1977: 118). For purposes of illustration, suppose a property structure consisting of only three properties: red,

\footnotetext{
${ }^{8}$ Carnap (1967: 125ff) further distinguishes between similarity (Sim), part-identity (Pi) and partsimilarity (Ps). We are not concerned with these distinctions, which are related to his choice of elementary experiences as the basic constituents of the construction system.

${ }^{9}$ This is the common mathematical term. As should be clear from the previous section, we are not committed to this mathematical characterization in our discussion of the notion of similarity.

${ }^{10}$ The term "similarity circle" hints at the geometrical conception underlying Carnap's construction. In a geometrical space in which similarity is determined by a metric, one indeed obtains circles or spheres by this method. The quasi-analytic method is not restricted to similarity structures on metrical spaces, but is applicable to any similarity structure. If one likes, one can also use the graph-theoretic term "maximal clique" (Leitgeb 2007: 187).
} 
blue, and yellow. Further suppose that our universe consists of precisely three discs, $d_{1}, d_{2}$, and $d_{3}$, the first of which is half red and half blue, the second half blue and half yellow, and the third half red and half yellow. Clearly, the three discs have none of our three properties in common, though each two of them do share a property. So, on Carnap's approach they are pairwise similar. As a result, quasi-analysis makes the set $\left\{d_{1}, d_{2}, d_{3}\right\}$ come out as being a property. However, ex hypothesi this property is not in our property structure, so that quasi-analysis has "recovered" a property that was not there to begin with, a spurious property, as one might say.

Whilst the above problems have convinced many philosophers that, at a minimum, similarity is a relation of little philosophical value, there is reason to believe that thereby these philosophers have overreacted. Already Carnap (1923, unpublished manuscript), who had discovered the companionship difficulty and the difficulty of imperfect community long before Goodman, believed that these difficulties were not disastrous, and had already explored solutions based on extra assumptions (see Carnap 1963: 946). Recently, Leitgeb (2007) and Mormann (2009) have presented precise mathematical analyses of the difficulties together with possible solutions.

Without going into technicalities, we would like to point at two philosophical consequences of Leitgeb's graph-theoretic construal of quasi-analysis. First, it becomes possible to formulate precise adequacy criteria that determine under which conditions quasi-analysis is an adequate procedure (Leitgeb 2007: 206). Unfortunately, the adequacy conditions Leitgeb formulates impose restrictions on the property structure that are unjustifiable and even metaphysically implausible. For instance, one of the conditions is that no property can be a subproperty of another property. In particular, it is stipulated that the unfavorable circumstances under which the companionship difficulty arises simply cannot occur. This view is hardly compatible with almost any philosophical theory of properties. Second, if one takes similarity as primitive and conceptually prior to the property structure, quasi-analysis always yields a unique and adequate property structure (Leitgeb 2007: 199). Goodman's problems only arise if one takes the property structure as primitive; for a large class of property structures, quasi-analysis cannot recover the original property structure on the basis of the similarity relation characterized by it. It should be stressed that Goodman's objections concerning the redundancy of similarity and the impossibility of defining properties presuppose a prior commitment to a given property structure. In view of this analysis, Leitgeb believes that, difficulties notwithstanding, quasi-analysis has a future.

Mormann (2009) offers a slightly different analysis. By means of additional requirements on the structure of (quasi-)properties-most importantly, that two elements that are similar to exactly the same elements have the same (quasi-)properties-he offers existence and uniqueness theorems for the quasianalytic method. He argues, pace Goodman, that in many philosophically relevant cases, this method can be fruitful.

Thus, recent work by Leitgeb and Mormann raises hope that quasi-analysis can be salvaged after all. At a minimum, this work warrants the conclusion that 
Goodman's arguments against the determination of properties on the basis of similarity are not as compelling as he thought them to be. But some might want to object to quasi-analysis on different grounds, dismissing it as being no more than philosophical fiction writing. "Indeed," it might be said, "have another look at the accounts you pointed at in arguing that a context-sensitive notion of similarity can be adequately defined. On these accounts, similarity plays no role in the constitution of properties. So, even if there is some philosophical method of reducing properties to similarities, psychologists appear to think it makes little contact with reality." That is not quite true, however.

Admittedly, it is true for Tversky's account, which makes properties prior to similarity: the contrast model already presupposes the existence of a domain of objects each of which is represented by a set of properties, or rather, for each context, it presupposes a domain of objects which are characterized by properties that are relevant in the given context. It thereby rules out from the start the construction of properties on the basis of the similarity relation.

However, that similarity plays no role in the constitution of properties is decisively not true on Gärdenfors's conceptual spaces account. Gärdenfors dismisses traditional accounts of properties. On his account, properties are identified with regions (sets of points) of conceptual spaces. The psychologically interesting properties - the "natural" properties-are identified with convex regions. ${ }^{11}$ So, for example, the property red is a convex region of color space. To arrive at a plausible account of categorization, that is, of how people carve up conceptual spaces into regions corresponding to natural properties, Gärdenfors invokes two further scientific concepts, to wit, prototypes and Voronoi tesselations. Prototype theory builds on the observation that among the instances of a property, some are more representative than others. The most representative one is the prototype of the property. A Voronoi tesselation of a given space divides that space into a number of cells such that each cell has a center and consists of all and only those points that lie no closer to the center of any other cell than to its own center; the centers of the various cells are called the generator points of the tesselation. Given that, by way of mathematical fact, Voronoi tesselations divide spaces into convex regions, a Voronoi tesselation of a conceptual space that takes points representing prototypes as generator points carves up that space into natural properties. In his book, and also in later articles, Gärdenfors musters some empirical evidence supporting the hypothesis that the foregoing account accords at least approximately with how people actually categorize reality. He also points to some theoretical considerations, in particular considerations of cognitive economy, that support the same conclusion (Gärdenfors 2000: 89).

If we accept that two objects $a$ and $b$ are similar tout court if they are similar in all respects or, more accurately perhaps, if they are similar in all contextually relevant respects, then Gärdenfors's account does not yield the

\footnotetext{
${ }^{11} \mathrm{~A}$ region is convex iff, for every pair of points in the region, the line between these points lies in its entirety in the region as well.
} 
neat reduction of properties to similarity relations that Carnap had envisioned. In fact, on that account, two objects may be similar without sharing so much as a single property. To see this, first note that, plausibly, on the conceptual spaces approach two objects may be said to be similar tout court if their distance in each of the contextually relevant conceptual spaces is below a certain threshold value. Now, it is possible that objects $a$ and $b$ are similar tout court, and therefore very close to each other in every relevant conceptual space, while, for each property $P$ in each relevant space $S$, object $a$ lies in $P$ while $b$ lies just outside $P .{ }^{12}$ Whether this will occur will be a contingent matter, depending on which respects are relevant, on the property structures of the conceptual spaces corresponding to the relevant respects, as well as on the similarity threshold or thresholds. Suppose, for instance, the only contextually relevant space is a onedimensional metric space consisting of the real number line with a Euclidean metric defined on it. Further, let property $P$ be represented by the interval (region) $\langle-\infty, 0]$, property $Q$ by the interval $[-1,1]$, and property $R$ by the interval $[0, \infty)$. Finally, let the similarity threshold be .1 . Then it is immediately clear that any two points that are similar tout court, and thus lie within a distance that is below the similarity threshold, share at least one property. Thus, in the present case, all similar objects share at least one property. Equally clearly, however, if we omit from the foregoing structure the property $Q$, then two objects may be similar without sharing any property.

However, it would be wrong to conclude that, on Gärdenfors's account as on Tversky's, similarity plays no role in the constitution of properties. Even if, on the former account, there is no simple reduction of properties to similarity, similarity plays a central role in it. Above, it was said that each cell of a Voronoi tesselation contains all and only those points of the given space that lie at least as close to the center of the cell as to the center of any of the other cells. The important point here is that closeness is measured by the similarity metric defined on the space at issue. So, that metric, together with the collection of points representing prototypes in the space, determines the property structure. That is not how Carnap thought similarity would help to constitute properties, but so what? All that matters for the purpose of justifying the claim that similarity is a philosophically useful notion, is that similarity does play a roleand a key one at that-in constituting properties.

In closing, we would like to note that, even quite independently of its potential role in determining the property structure, similarity may make itself useful as a philosophical tool in a number of ways. One such independent but philosophically interesting application is to be found in Hampton's work on vagueness - a philosophically highly relevant notion - that explicates vagueness in terms of graded set-membership, which in turn is explained in terms of similarity (Hampton 1998, 2007). ${ }^{13}$ Another is to be found in Douven and

\footnotetext{
${ }^{12}$ To be entirely precise, we should say that $a$ is represented by a point in the region corresponding to $P$ in $S$, but we trust that no confusion will arise from our looser way of speaking.

${ }^{13}$ See Douven et al. (2010) for an alternative account of vagueness in terms of similarity.
} 
Decock (2010), where it is argued that the so-called paradoxes of identitypuzzle cases involving the possibility of change over time and issues of constitution - can be explained in a uniform and elegant manner by construing the identity predicate as it occurs in those paradoxes in terms of similarity. Neither of these applications of the notion of similarity assumes that properties can be defined on the basis of a similarity relation, whether via quasi-analysis or via some other procedure. A third case in point, this one from the philosophy of science, is the role attributed to similarity in the so-called semantic conception of theories. For a long time, analytic philosophers had thought of scientific theories as (typically, axiomatizable) sets of sentences, much like mathematical theories. But in the 1970s and 1980s several philosophers argued for a different view according to which theories are to be conceived as sets of models together with claims about how the given models relate to the world. Originally, the relation to the world was spelled out in terms of isomorphism (see, e.g., van Fraassen 1980: 64-69). However, Giere (1988, Ch. 3) forcefully argued that the requirement of isomorphism is too strong and would fail to do justice to many scientific theories. The appropriate model-world relation, according to him, is rather one of similarity in certain respects. ${ }^{14}$ Meanwhile, this has become almost common lore among philosophers of science.

To summarize, largely due to Goodman's influential critique of the notion of similarity, most philosophers have taken a dim view of that notion. But while Goodman's critique effectively highlights some shortcomings of the model of similarity that was dominant at the time when the critique was written, it shows little about the notion of similarity per se. As was seen, since Goodman's critique, accounts of similarity have been put forth that are impervious to the arguments given in that critique. We focused on what we take to be the two best such accounts, to wit, Tversky's and Gärdenfors's, and we argued that on neither of these is similarity a slippery notion. To the contrary, both provide a formally precise definition of similarity that takes into consideration the context-sensitivity of, and asymmetry in, people's similarity judgments. Moreover, we have argued that Goodman's companionship difficulty and his difficulty of imperfect community need not undermine the method of quasianalysis, whereby properties could be distilled from similarities. Even if it did, assuming the said method is not necessary for viewing similarity as a philosophically important relation, provided Gärdenfors's conceptual spaces approach is correct. In short, similarity is a scientifically kosher notion that also belongs in the philosopher's toolkit, even though it may not be exactly the tool that philosophers before Goodman's critique believed it to be. ${ }^{15}$

\footnotetext{
${ }^{14}$ Giere does not specify the notion of being similar in certain respects. In particular, it is not clear whether he would opt for Tversky's or Gärdenfors's explication of similarity (or even for some third).

${ }^{15} \mathrm{We}$ are greatly indebted to Christopher von Bülow and to two anonymous reviewers for this journal for very helpful comments on earlier versions. Thanks also for useful discussion to the participants of a research meeting at the VU University Amsterdam.
} 
Open Access This article is distributed under the terms of the Creative Commons Attribution Noncommercial License which permits any noncommercial use, distribution, and reproduction in any medium, provided the original author(s) and source are credited.

\section{References}

Carnap, R. 1923. Die Quasizerlegung: Ein Verfahren zur Ordnung nichthomogener Mengen mit den Mitteln der Beziehungslehre. Unpublished manuscript RC-081-04-01, University of Pittsburgh.

Carnap, R. 1928/1967. The logical structure of the world. Berkeley: University of California Press.

Carnap, R. 1963. Nelson Goodman on Der logische Aufbau der Welt. In The philosophy of Rudolf Carnap. ed. P.A. Schilpp, 944-947. La Salle: Open Court.

Clark, A. 1993. Sensory qualities. Oxford: Clarendon.

Coombs, C.H. 1954. Method for the study of interstimulus similarity. Psychometrika 19: 183-194.

Decock, L. 2006. A physicalist reinterpretation of 'phenomenal' spaces. Phenomenology and the Cognitive Sciences 5: 197-225.

Douven, I., and L. Decock. 2010. Identity and similarity. Philosophical studies. In press.

Douven, I., L. Decock, R. Dietz, and P. Égré. 2010. Vagueness: A conceptual spaces approach. Manuscript.

Gärdenfors, P. 2000. Conceptual spaces. Cambridge: Bradford.

Giere, R. 1988. Explaining science. Chicago: Chicago University Press.

Goldstone, R.L., and J.Y. Son. 2005. Similarity. In The Cambridge handbook of thinking and reasoning, ed. K.J. Holyoak, and R.G. Morrison, 13-36. Cambridge: Cambridge University Press.

Goodman, N. 1965. Fact, fiction, and forecast. New York: Bobbs-Merrill.

Goodman, N. 1972. Seven strictures on similarity. In Problems and projects, 437-446. Indianapolis/ New York: Bobbs-Merrill.

Goodman, N. 1977. The structure of appearance. Dordrecht: Reidel.

Goodman, N. 1941/1990 A study of qualities. New York: Garland.

Hampton, J.A. 1998. Similarity-based categorization and fuzziness of natural categories. Cognition 65: $137-165$.

Hampton, J.A. 2007. Typicality, graded membership, and vagueness. Cognitive Science 31: 355384.

Holman, E.W. 1979. Monotonic models for asymmetric proximities. Journal of Mathematical Psychology 20: 1-15.

Johannesson, M. 2000. Modelling asymmetric similarity with prominence. British Journal of Mathematical and Statistical Psychology 53: 121-139.

Krumhansl, C.L. 1978. Concerning the applicability of geometric models to similarity data: The interrelationship between similarity and spatial density. Psychological Review 85: 445-461.

Leibniz, G.W. 1923. Sämtliche Schriften und Briefe. Berlin: Akademie Verlag.

Leitgeb, H. 2007. A new analysis of quasianalysis. Journal of Philosophical Logic 36: 181-226.

Medin, D.L., R.L. Goldstone, and D. Gentner. 1993. Respects for similarity. Psychological Review 100: 254-278.

Meijs, W. 2005. Probabilistic measures of coherence. Doctoral dissertation, Erasmus University Rotterdam.

Mormann, T. 2009. New work for Carnap's quasi-analysis. Journal of Philosophical Logic 38: 249-282.

Nosofsky, R.M. 1991. Stimulus bias, asymmetric similarity, and classification. Cognitive Psychology 23: 94-140.

Quine, W.V.O. 1969. Natural kinds. In his Ontological relativity and other essays, 114-138. New York: Columbia University Press.

Shepard, R.N. 1958. Stimulus and response generalization: Tests of a model relating generalization to distance in psychological space. Journal of Experimental Psychology 6: 509-523.

Shepard, R.N. 2001. Perceptual-cognitive universals as reflections of the world. Behavioral and Brain Sciences 24: 581-601. 
Torgerson, W.S. 1958. Theory and methods of scaling. New York: Wiley.

Torgerson, W.S. 1965. Multidimensional scaling of similarity. Psychometrika 30: 379-393.

Tversky, A. 1977. Features of similarity. Psychological Review 84: 327-354.

Tversky, A., and I. Gati. 1982. Similarity, separability, and the triangle inequality. Psychological Review 89: 123-154.

van Fraassen, B.C. 1980. The scientific image. Oxford: Clarendon Press. 